\title{
Angular dependence of the luminance and contrast in medical monochrome liquid crystal displays
}

\author{
Aldo Badano ${ }^{\text {a) }}$ \\ Center for Devices and Radiological Health, Food and Drug Administration, 12720 Twinbrook Parkway, \\ Rockville, Maryland 20857 \\ Michael J. Flynn \\ Department of Radiology, Henry Ford Health System, Detroit, Michigan 48202
}

\begin{abstract}
Sandrine Martin and Jerzy Kanicki
Department of Electrical Engineering and Computer Science, University of Michigan, Ann Arbor, Michigan 48109
\end{abstract}

(Received 28 August 2002; accepted for publication 10 July 2003; published 17 September 2003)

Active-matrix liquid crystal displays (AMLCDs) are light-modulating devices that generate images by differentially transmitting a nearly uniform luminous field provided by a backlight. While emissive displays exhibit a quasi-Lambertian emission with almost constant contrast at off-normal viewing, the anisotropy of the electro-optic effect that controls light transmission in AMLCDs causes a pixel luminance that varies, sometimes strongly, with viewing angle. These variations are not identical for all gray levels and can eventually cause grayscale inversions. In this paper, we measured the luminance emission of a monochrome medical AMLCD, a medical cathode-ray tube monitor, and a color desktop AMLCD, using a collimated photopic probe positioned on a manual rotation arm, and a research radiometer with automatic readout. The probe measures luminance with a small acceptance angle and provides optical shielding from emissions at other viewing directions that contaminate the readings. We obtained luminance response curves versus angle in the vertical, horizontal and at $45^{\circ}$ diagonal directions. The display systems were calibrated to reflect the DICOM Part 3.14 standard grayscale display function (GDF) when measured using the manufacturer's probe and software tools. We analyzed the measurements at different viewing directions with respect to their departure from the GDF by computing the normalized contrast $(\Delta L / L)$ as a function of the DICOM just-noticeable difference index. Although cathode-ray tubes are known to be quasi-Lambertian emitters, the luminance at normal viewing is higher than the luminance observed at large angles. This decrease in luminance is however proportionally similar for all gray levels, resulting in a relatively flat contrast response for all angles. In addition to being more pronounced, the angular variation in AMLCDs does not follow the same profile at different intensities with the subsequent variation in the achieved display contrast. The changes due to off-normal viewing are substantial at large angles in the horizontal and vertical directions, and much worse in the diagonal viewing directions. () 2003 American Association of Physicists in Medicine.

[DOI: $10.1118 / 1.1606449$ ]

Key words: liquid crystal display, medical display, luminance response, display contrast, viewing angle

\section{INTRODUCTION}

Digital imaging systems used in radiology rely on electronic display devices to present images to human observers. For available devices, the electronic display of digital radiographs entails some degradation of image quality as compared to the quality of a trans-illuminated film on a viewbox. However, we know that the detection and classification of subtle abnormal conditions in trans-illuminated radiographs is limited by the performance of the human visual system. Flynn et al. ${ }^{1}$ recognized that fact, and defined the performance of a high fidelity display that matches the capabilities of human vision. It was also shown in Ref. 1 that current electronic displays do not provide the quality required for a high fidelity presentation of digital radiographic images, due to a number of reasons that include insufficient spatial resolution, veiling glare, and excessive reflections.

An important characteristic of a display device is the angular luminance distribution. Some applications, such as electronic cashier consoles and back-seat entertainment monitors for airplanes, benefit from a narrow angular luminance to prevent spectators from visualizing the information presented to the primary viewer. In radiology, however, common situations involve collective viewing of a diagnostic image by several persons. Examples of this include a group of radiology residents or two specialists discussing a specific case that is displayed on a workstation monitor.

Ideally, the luminance of a display device should not vary as a function of the viewing angle. This occurs for devices where the emission of visible light (i.e., the luminous inten- 
sity in lumens/sr) from the display surface varies with the cosine of the angle from the surface normal vector, $I(\theta)$ $=I_{0} \cos \theta$, where $I_{0}$ is the intensity at $\theta=0$. Since luminance is proportional to the light emitted per unit surface area projected along a particular direction given by $A_{0} \cos \theta$, the luminance is equal to $I_{0} / A_{0}$ and independent of $\theta$. Light emitting surfaces of this type are referred to as having a Lambertian emission since the emission follows Lambert's cosine law.

The angular luminance distribution of cathode-ray tubes (CRTs) is quasi-Lambertian. ${ }^{2}$ In CRTs, light patterns are generated by the raster scan of a high-energy electron beam. The electron energy is converted to light photons in a cathodoluminescent phosphor within the CRT emissive structure. The angular distribution of light emitted by cathodoluminescence can be considered to be isotropic. Then, before exiting through the faceplate, photons undergo a large number of scattering events in the granular phosphor layer, at the $\mathrm{Al}$ reflective backing film, and within the thick glass faceplate. In the presence of a reflective surface on one side, this light scattering process (that originated with an isotropic emission within the phosphor), results in a Lambertian-like angular emission. Similar angular emission profiles have been documented for other light-emitting display technologies with analogous emissive structures where isotropic light is generated in a thin-film layer sandwiched between a reflective backing and a front transparent faceplate. ${ }^{3}$ Light absorption in the glass faceplate introduced to reduce veiling glare and control ambient reflections can cause some degree of departure from the Lambertian profile, especially at large viewing angles due to longer paths through the absorptive faceplate.

In the case of liquid crystal displays (LCDs), the manner in which light patterns are generated establishes an angular emission that is far from Lambertian. In LCDs, images are formed by modulating the transmittance of a uniform backlight through a liquid crystal cell. The electro-optic effect responsible for the modulation of the light intensity takes place in a few microns of liquid crystal material sandwiched between substrates, and in polarization films, alignment layers, and other optical coatings and layers. The voltage applied across the LC material controls the light transmission through the LC cell (i.e., the pixel luminance) by determining the spatial configuration of the LC molecular arrangement. Light is polarized by a first filter and, as it passes through the LC layer, experiences a twist in its polarization direction. A second polarizer in the front substrate selectively transmits light with the modified polarization state. The pixel luminance is affected by two factors: (a) by the path length across the cell, and (b) by the relative orientation of the light photon polarization direction and the liquid crystal molecules. Both of these factors vary in a complex manner for paths traversing the LC cell at different angles with respect to the display normal (see Fig. 1).

The design of LCD structures is typically optimized for normal viewing with contrast changes occurring at offnormal viewing directions. During the last 10 years, significant improvements have been made to devise LCD structures with improved viewing angle performance, i.e., where the

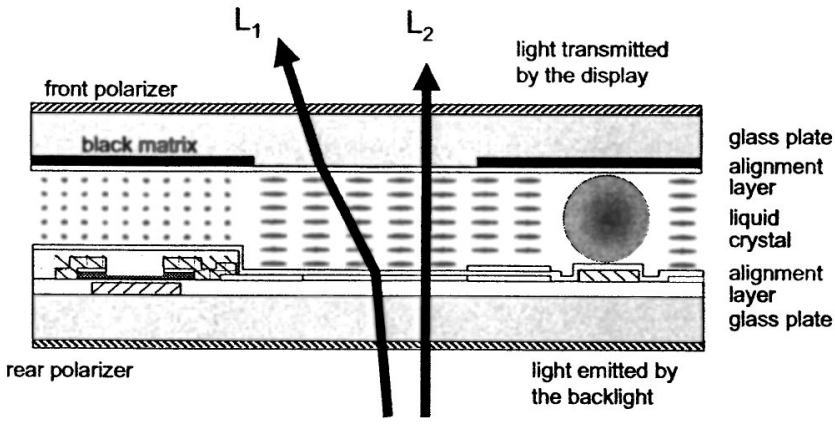

FIG. 1. Due to the anisotropic electro-optic effect that these two light paths experience, the luminance outputs $L_{1}$ and $L_{2}$ are not equal, even though they come from the same pixel. The resulting emission from the AMLCD is far from Lambertian.

image quality observed normally to the display surface normal is maintained at off-normal viewing directions over a wider cone. The most commonly employed design concepts used to achieve a wider angular emission are multiple domains, ${ }^{4-9}$ modified LC configurations (including in-plane switching ${ }^{10,11}$ and vertically aligned molecules ${ }^{12}$ ) and compensation films. ${ }^{13,14}$ Devices with good viewing angle performance often have a combination of these features in their LC structures.

In this paper, we report the luminance response (luminance output versus the display controller gray level) at varying viewing angles for a medical monochrome active-matrix LCD (AMLCD) monitor marketed for digital radiology applications. We compare the results obtained for the medical AMLCD to the response obtained for a medical monochrome CRT, and for a desktop color AMLCD monitor. Luminance measurements were made using two different methods. The first method used a manual rotation arm and a collimated photopic probe. The second method used Fourier optics and a CCD camera to map the recorded luminance to angular intensity values. Our luminance measurements are described in terms of changes in the grayscale display function (GDF). In addition, we present the same data expressed as normalized contrast per just-noticeable-difference (JND) interval, as a more sensitive metric to study the departure from the desired GDF.

\section{METHODS}

In this paper, we investigate the viewing angle characteristics of three display systems. The first is the C3 from PLANAR Systems, Inc. (Beaverton, OR), a medical monochrome AMLCD monitor with 3 million $(1536 \times 2048)$ pixels having a dual-domain, in-plane switching design. The monitor was attached by a digital video interface to a MD5 driver board (also from PLANAR Systems. The screen size is $528.3 \mathrm{~mm}$ in diagonal $(485.9 \mathrm{~mm} \times 381 \mathrm{~mm})$. Although flat-panel was originally designed for full-color applications, ${ }^{15}$ this particular product has no color filters (color in AMLCDs is achieved by selective filtering of the broad spectrum emitted by the backlight). This implies that in the monochrome version, each pixel consists of three sub-pixel regions associated 


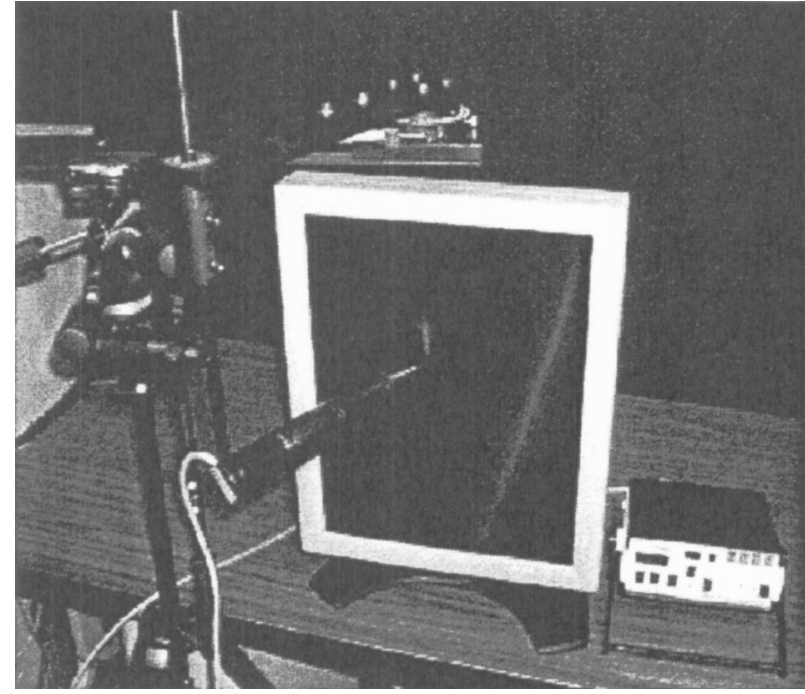

FIG. 2. Experimental setup to measure luminance as a function of the viewing direction. The rotation arm and collimated probe allow one to measure luminance coming from a small spot in the screen along an arc, maintaining the same distance between the probe and the spot at all angles.

with the red, green, and blue color pixels. The removal of the color filters leads to an achievable maximum luminance for the monochrome version of about twice the maximum luminance of the equivalent color monitor for the same backlight configuration. The second system is a 5 million (2048 $\times 2560$ ) pixel monochrome CRT in portrait mode with P45 phosphor driven by a 5MP board (BARCO Medical Displays, Duluth, GA). The viewable area of the CRT display is $481 \mathrm{~mm} \times 377 \mathrm{~mm}$. Finally, the third display system measured in this study is a 1.3 million $(1280 \times 1024)$ pixel active-matrix liquid crystal color monitor for desktop applications (SAMSUNG SyncMaster TFT800) with a display area of $359.0 \mathrm{~mm} \times 287.2 \mathrm{~mm}$ and a pixel pitch of $0.281 \mathrm{~mm}$, driven by a MATROX Millennium G400 video board.

All the display systems used in this study were calibrated to DICOM 3.14 GDF via software and a measuring probe used in proximity to the display faceplate. The medical AMLCD was calibrated using DOME's TQA software, while the monochrome CRT and color AMLCD were calibrated using the VERILUM software (IMAGESMITHS Inc., Gaithersburg, MD). The medical CRT was calibrated with a luminance range of 600 , from a minimum luminance of $0.2 \mathrm{~cd} / \mathrm{m}^{2}$ to a maximum of $120 \mathrm{~cd} / \mathrm{m}^{2}$. The medical AMLCD system was calibrated in three alternative regimes: an extended luminance range of 800 from 1.0 to $800 \mathrm{~cd} / \mathrm{m}^{2}$, and two regimes with a reduced luminance range of 200 . For the reduced luminance range, we used minimum luminance values of 2 and $4 \mathrm{~cd} / \mathrm{m}^{2}$, with the corresponding maximum luminance of 400 and $800 \mathrm{~cd} / \mathrm{m}^{2}$. The color AMLCD was calibrated within a narrower range, from 0.5 to $100 \mathrm{~cd} / \mathrm{m}^{2}$.

We measured the angular luminance profiles using two different methods. The first method used small-spot luminance measurements made with a conic collimated photopic probe positioned with a manual rotation arm (see Fig. 2). The conic probe measures luminance with a small field-of-view

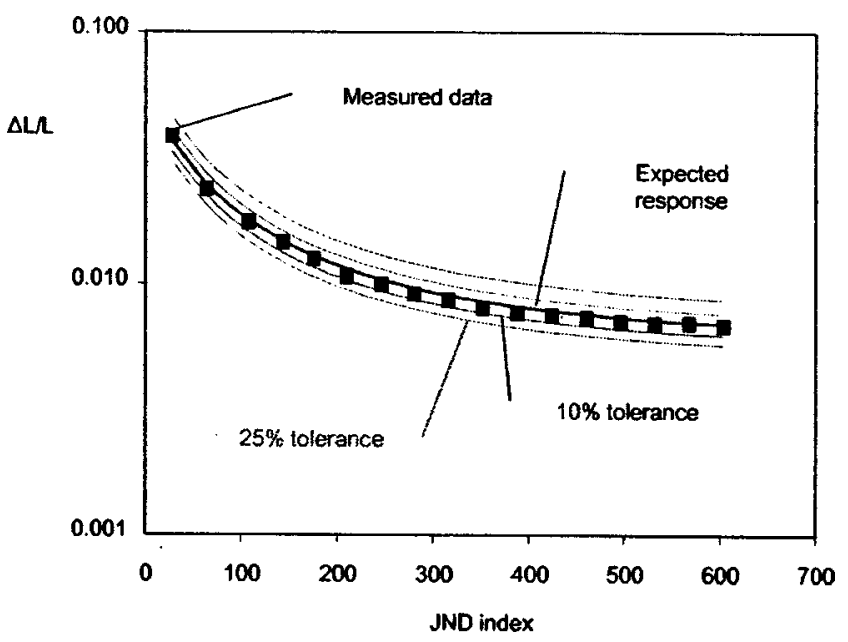

FIG. 3. Example of the normalized contrast $(\Delta L / L)$ representation of the angular data as a function of the JND index. The squares represent the experimentally measured data points. The solid line depicts the expected response for a DICOM-compliant system. The dashed (dotted) lines indicate the $10 \%(25 \%)$ tolerance limits.

and provides optical shielding from emissions at other viewing directions that can contaminate the readings. ${ }^{16}$ This is especially critical at large off-normal angles where the luminance measurement would be corrupted by light coming from regions of the display that are closer to the probe (far away from the desired measurement spot), representing a completely different viewing direction with respect to the one intended. The probe was connected to a research radiometer with a serial line computer interface that allowed measured luminance to be recorded by a software application that displays one of the 256 gray levels in a square target (10\% area of the full field) with a constant background ( $20 \%$ of the maximum luminance). Eighteen consecutive luminance measurements were acquired for test regions at gray levels varying from 0 to 255 in steps of 15 . Each measured luminance value was obtained by averaging 10 observations made with a $0.5 \mathrm{~s}$ integration time. Angular emission distribution profiles were acquired for the vertical and horizontal directions, and for $45^{\circ}$ diagonal directions. The results were analyzed with respect to their departure from the DICOM Part $3.14 \mathrm{GDF}^{17}$ by computing the normalized contrast as a function of the JND index and plotting the experimental results along with the expected response with $10 \%$ and $25 \%$ tolerance limits (see Fig. 3). We chose to include the curves corresponding to these tolerance limits because they are being considered by the AAPM Task Group number $18^{18}$ as recommended values for the acceptance testing and clinical quality control of medical display devices.

The expected response was computed from the luminance values associated with the DICOM Standard Display Function, page 16, Annex B (Ref. 17). The contrast metric $(\Delta L / L)$ was calculated for both the expected and the measured response as the corresponding slope, i.e., $\Delta \log (L) / \Delta$ JND, where $\Delta$ indicates the difference between two consecutive data points.

The second method relies on Fourier optics to map lumi- 


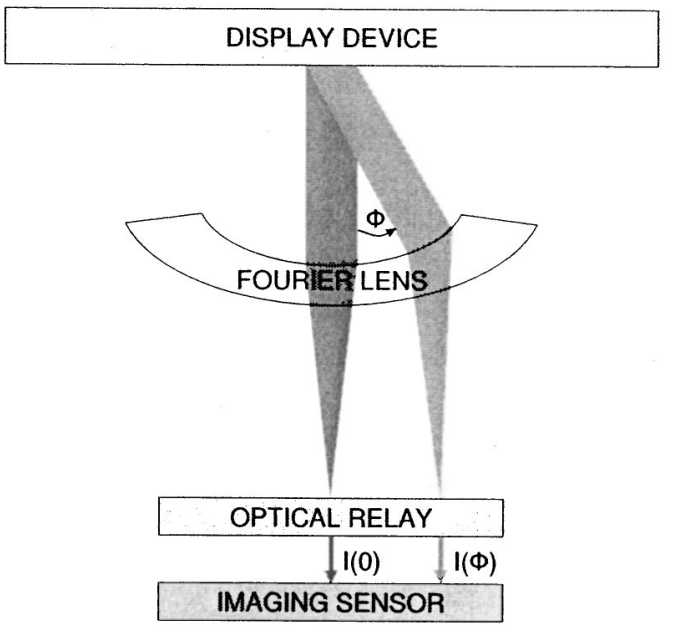

FIG. 4. Schematic of the Fourier optics method for measuring angular luminance distributions. The luminous intensity $I(0)$ is mapped onto the imaging sensor at the center of the array, while the off-normal intensity $I(\phi)$ hits the sensor at a location away from the center.

nance intensity to angular luminance using a cooled CCD. ${ }^{19}$ We used a commercial system (EZContrast 160D) mounted on a motorized stage (EZMotion), manufactured by ELDIM



(a)

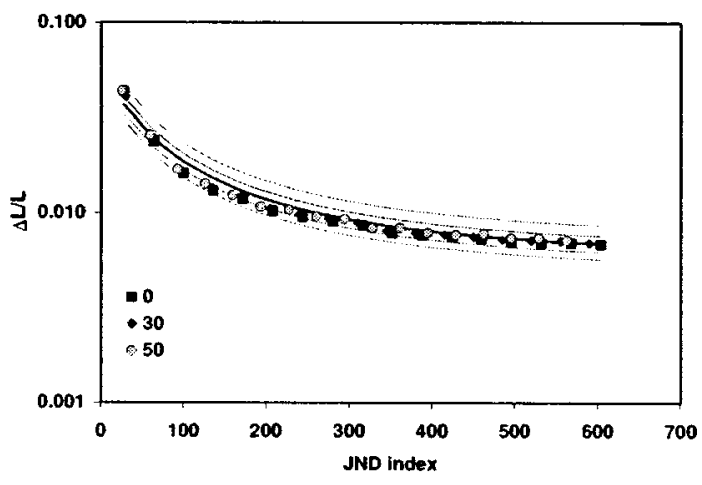

(b)

FIG. 5. Viewing angle characteristics of the medical CRT. (a) The luminance output as a function of gray level is plotted for selected off-normal angles. The same data are presented in (b), analyzed in terms of contrast per JND index. See Fig. 3 for details on this representation.
TABLE I. Luminance reduction factor $L_{f}(\phi)$ for the medical CRT and AMLCD calculated from the luminance measurements $(L)$ at different viewing angles.

\begin{tabular}{|c|c|c|c|c|c|c|}
\hline \multirow[b]{2}{*}{ Display device } & \multirow[b]{2}{*}{ Gray level } & \multicolumn{2}{|c|}{$L_{f}(\phi)$} & \multicolumn{3}{|c|}{$L\left(\mathrm{~cd} / \mathrm{m}^{2}\right)$} \\
\hline & & $30^{\circ}$ & $45^{\circ}$ & $0^{\circ}$ & $30^{\circ}$ & $45^{\circ}$ \\
\hline \multirow[t]{3}{*}{ CRT } & 15 & 0.90 & 0.90 & 0.80 & 0.72 & 0.65 \\
\hline & 135 & 0.91 & 0.88 & 34.8 & 31.6 & 27.8 \\
\hline & 255 & 0.91 & 0.88 & 283 & 257 & 226 \\
\hline \multirow{3}{*}{$\begin{array}{c}\text { AMLCD } \\
\text { (horizontal) }\end{array}$} & 15 & 0.94 & 0.91 & 2.53 & 2.38 & 2.16 \\
\hline & 135 & 0.86 & 0.79 & 84.5 & 72.9 & 57.8 \\
\hline & 255 & 0.81 & 0.74 & 821 & 668 & 496 \\
\hline \multirow{3}{*}{$\begin{array}{l}\text { AMLCD } \\
\text { (vertical) }\end{array}$} & 15 & 1.40 & 1.06 & 2.53 & 3.53 & 3.73 \\
\hline & 135 & 0.77 & 0.69 & 81.6 & 62.8 & 43.3 \\
\hline & 255 & 0.76 & 0.67 & 801 & 612 & 413 \\
\hline \multirow{3}{*}{$\begin{array}{c}\text { AMLCD } \\
\text { (diagonal 1) }\end{array}$} & 15 & 2.71 & 2.13 & 2.55 & 6.91 & 14.7 \\
\hline & 135 & 0.87 & 0.78 & 84.2 & 72.9 & 56.7 \\
\hline & 255 & 0.78 & 0.60 & 812 & 634 & 379 \\
\hline \multirow{3}{*}{$\begin{array}{c}\text { AMLCD } \\
\text { (diagonal 2) }\end{array}$} & 15 & 1.92 & 2.56 & 2.52 & 4.83 & 12.4 \\
\hline & 135 & 0.85 & 0.77 & 83.1 & 70.6 & 54.6 \\
\hline & 255 & 0.79 & 0.61 & 803 & 634 & 385 \\
\hline
\end{tabular}

(Hérouville St. Clair, France). This method utilizes a Fourier lens and a cooled CCD sensor. The lens provides a Fourier transform image of the display surface emission. Every light beam emitted from the display test area with an angle $\phi$ (see Fig. 4) is focused on the focal plane at a relative position with respect to the center of the test area that depends only on $\phi$. There exists a one-to-one correspondence between a direction of emission (or viewing direction), and the intensity at specific spatial locations in the imaging sensor. An optical relay system scales the Fourier transform image at the measured surface on the CCD sensor. The viewing angle map is obtained by processing the acquired image with appropriate calibration functions provided by the manufacturer. Since all of the angular information is obtained by a single imaging sensor through the Fourier lens, no rotation of the measuring device or display unit is required. Although the diagram shown in Fig. 4 is two dimensional, the instrument can measure angular luminance in the entire hemisphere (up to $80^{\circ}$ from the display surface normal). Measurements were performed at different gray levels using large centered targets and a measuring spot size of $2 \mathrm{~mm}$. We used $0.5^{\circ}$ angular steps for both polar and azimuth angles. Iso-luminance and iso-contrast plots were obtained for each measured gray level.

Another useful way to analyze angular emission profiles of display devices is to calculate a factor that correlates with the magnitude of the departure from an ideal Lambertian emission. In this paper, we introduce the luminance reduction factor $L_{f}$. The factor is calculated for each viewing angle as follows:

$$
L_{f}(\phi)=L(\phi) / L(0) \text {, }
$$

where $L(\phi)$ is the luminance measured at an angle $\phi$ from the display surface normal along a specific orientation (horizontal, vertical, diagonal), and $L(0)$ is the luminance at $\phi$ $=0^{\circ}$. A factor equal to one for all angles represents a display 


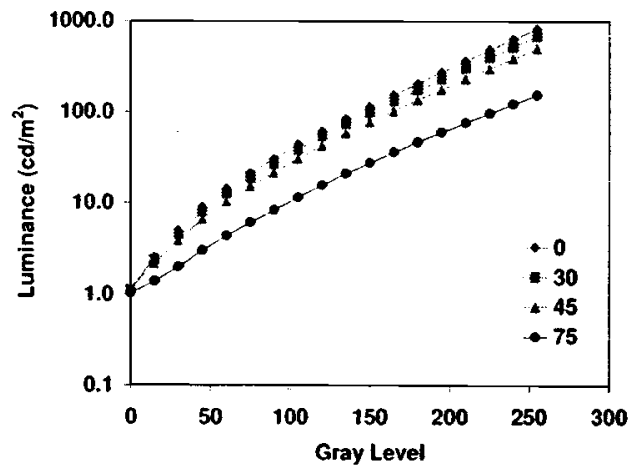

(a)



(c)

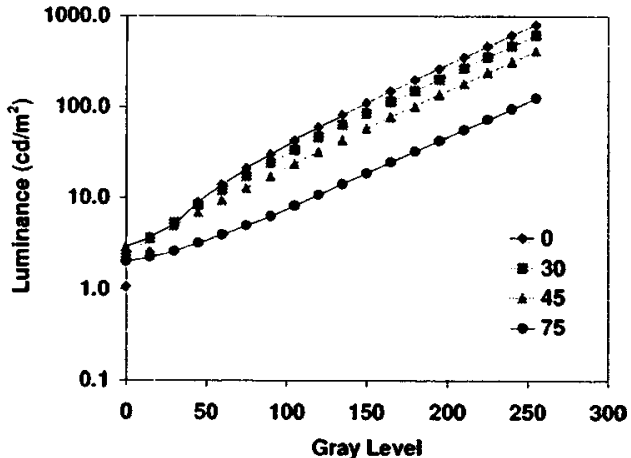

(b)

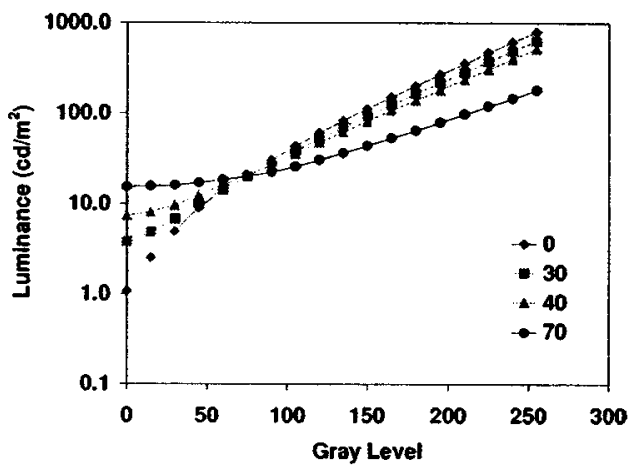

(d)

FIG. 6. Angular luminance profiles for the medical AMLCD in the extended luminance range regime. The four plots show the luminance output as a function of gray level for selected off-normal angles in the horizontal (a), vertical (b), and diagonal (c) and (d) directions.

emission with constant luminance, typical of Lambertian surfaces. Values of $L_{f}$ greater than one depict emissions where the luminance increases with off-normal angle, while $L_{f}$ values smaller than one are representative of forward-peaked emissions.

All measurements, with the exception of those taken with the Fourier system, were done in a display laboratory with absorptive flat black walls and black ceiling and floor, to reduce any contamination of the measurements by light reflection. The measurements with the Fourier optics method were done in a room with controlled illumination.

\section{RESULTS}

Figure 5 shows the angular luminance and contrast response of the CRT monitor. Figure 5(a) confirms that the CRT emission resembles a Lambertian profile. The values of $L_{f}$ obtained for the CRT are presented in Table I. In the case of the CRT, the $L_{f}$ values for the angles considered in this calculation $\left(30^{\circ}\right.$ and $\left.45^{\circ}\right)$ are on the order of 0.90 . Due to the isotropic character of the angular luminance distribution, we show data only for the horizontal direction. Although there is a change in overall magnitude of the luminance response, the contrast response demonstrates almost no change with off-normal angle.
In Figs. 6 and 7, we present the data for the medical monochrome AMLCD operated over the full luminance range. The results are plotted separately for the horizontal, the vertical, and the two diagonal directions (from bottomleft to top-right and from bottom-right to top-left). The luminance response of the monochrome AMLCD changes varies with viewing angle notably in the low luminance range, especially in the diagonal directions. The minimum measured luminance increases by a factor of about 10 when the viewing direction moves along the oblique axes. The values of $L_{f}$ obtained for the medical monochrome AMLCD are presented in Table I for comparison with the CRT data. The values of the luminance reduction factor, indicative of how the system behaves in comparison with a Lambertian emitter, show a consistent trend of larger departures at low gray levels (15 in Table I), and at diagonal directions. In the case of the medical monochrome AMLCD, the $L_{f}$ values vary significantly with angle and orientation. The maximum departure from Lambertian, indicated by the maximum $L_{f}$ occurs at the gray level of 15 at $30^{\circ}$ along the diagonal orientation 1 (corresponding to the direction from bottom-left to top-right) with a $L_{f}$ value equal to 2.71 . The departure from Lambertian is seen also at high luminance (high gray level) where $L_{f}$ 


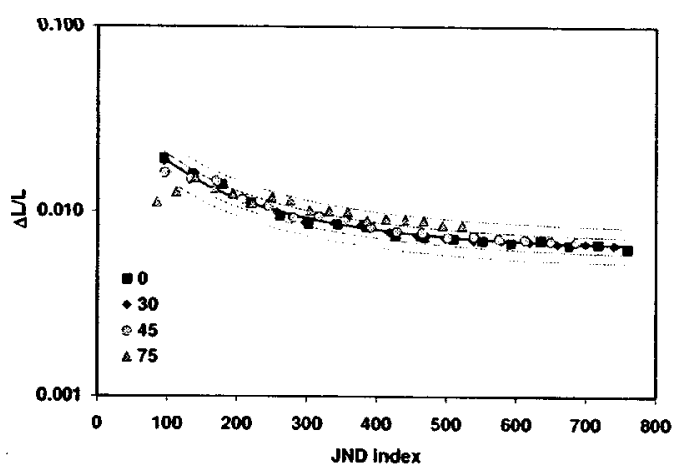

(a)

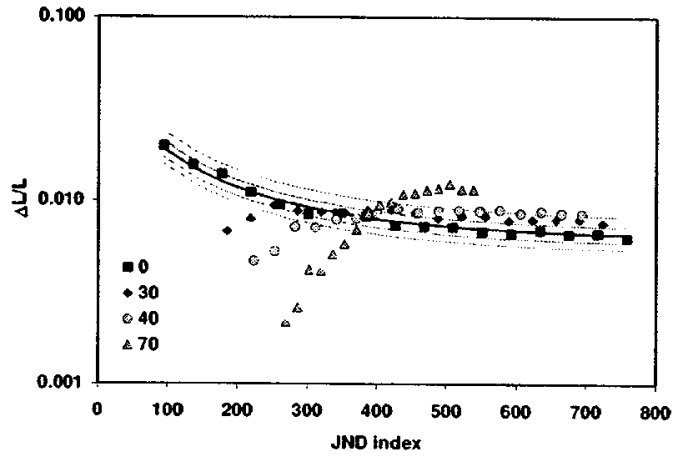

(c)



(b)

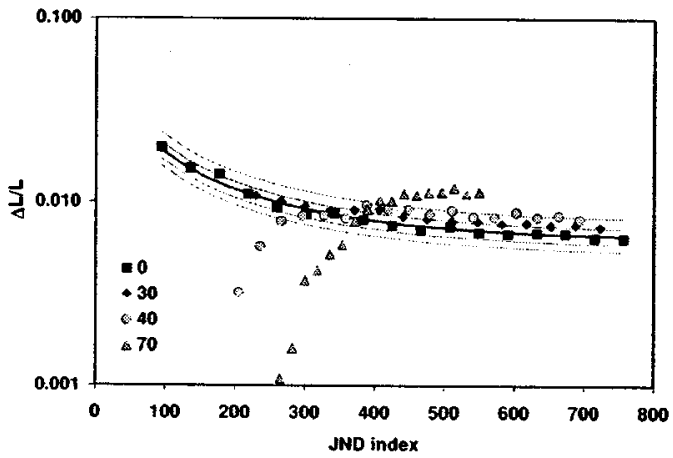

(d)

FIG. 7. The same data presented in Fig. 6, analyzed in terms of normalized contrast per JND. Also, (a) represents data along the horizontal direction, (b) along the vertical, and (c) and (d) along the diagonal directions.

values as small as 0.60 were obtained (for the diagonal 1 at $45^{\circ}$ ).

In addition, we observe that the slope of the curve in Fig. 6 , which is associated with image contrast, is significantly reduced in the low luminance region. This is confirmed by analyzing the contrast response plots in Fig. 7. The available contrast per JND decreases rapidly when the observer moves along the diagonal directions. Even at a relatively small angle of $30^{\circ}$, the contrast response in the low luminance region falls outside of the $25 \%$ tolerance limits.

Figures 8 and 9 show the results for the medical AMLCD, using a reduced luminance range of 200. In this case, we observe that the deviation from the normal measurements is more severe for the system calibrated at low luminance $\left(2-400 \mathrm{~cd} / \mathrm{m}^{2}\right)$ than for the system with calibration at higher luminance $\left(4-800 \mathrm{~cd} / \mathrm{m}^{2}\right)$.

All results presented up to this point were measured with the collimated probe and rotation arm. Figure 10 shows isoluminance plots of the display luminance in the white (a) and black (b) states measured with the Fourier optics method. We can see that the AMLCD maximum luminance exhibits circular symmetry. It decreases at off-normal viewing angles, but remains about $200 \mathrm{~cd} / \mathrm{m}^{2}$, or $35 \%$ of the luminance in the normal direction at an angle of $75^{\circ}$. In the black state, the luminance changes are relatively small along the horizontal and vertical directions, but there is a severe increase of the black luminance along the display diagonals, as expected for an AMLCD based on in-plane switching technology. ${ }^{20}$ For an off-normal angle of $75^{\circ}$, the AMLCD minimum luminance increases from about $1.5 \mathrm{~cd} / \mathrm{m}^{2}$ to more than $10 \mathrm{~cd} / \mathrm{m}^{2}$ along the diagonals. Consequently, the AMLCD contrast ratio is more severely degraded along the diagonals than in the horizontal and vertical directions, as seen in Fig. 10(c).

The results obtained using the Fourier optics method are in good agreement with the results from the rotating probe measurement method, as demonstrated in Fig. 11. The two methods resulted in practically the same results for gray levels greater than 60. For small gray levels, the results obtained with the collimated probe are about $5 \%$ higher than those obtained with the Fourier optics system.

Finally, Figs. 12 and 13 show the luminance and contrast response for the color desktop AMLCD. Note that because of the limitations in the flat-panel and driver boards, the luminance response spans only 400 JNDs at normal viewing. It remains useful for us to compare the performance of this display system with the performance of the monochrome AMLCD. We observe that the contrast at low luminance increases with viewing angle. On the other hand, the contrast at high JNDs is smaller at off-normal angles than for the normal viewing direction. This behavior is opposite to what 




(a)

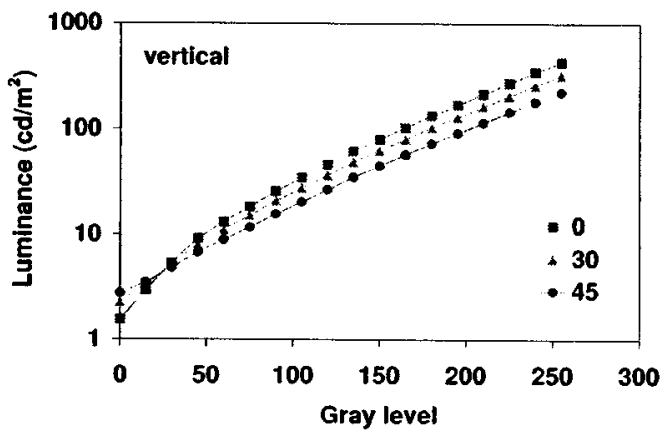

(c)

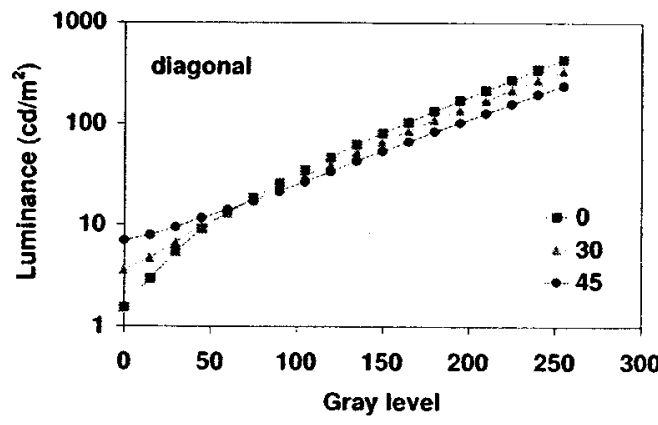

(e)

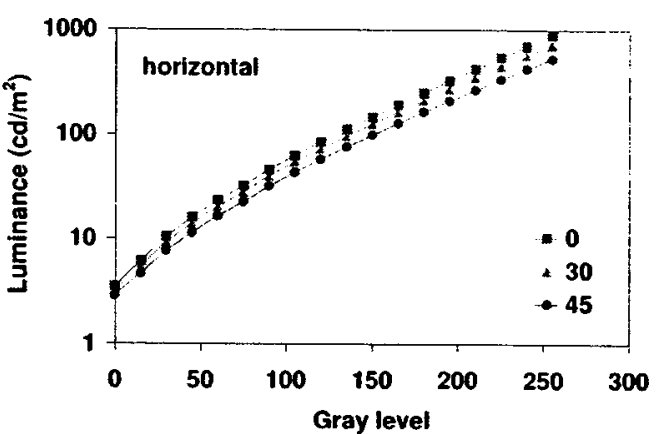

(b)

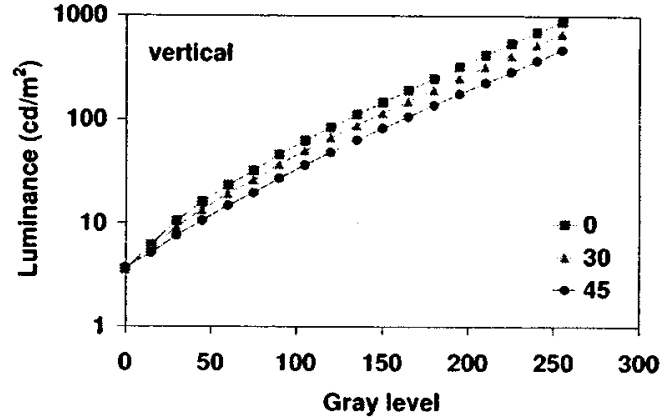

(d)



(f)

FIG. 8. Angular luminance profiles for the medical AMLCD in the reduced luminance range regime. The six plots show the luminance output as a function of gray level for selected off-normal angles in the horizontal [(a) and (b)], vertical [(c) and (d)], and diagonal [(e) and (f)] directions. The two columns correspond to the two luminance levels utilized for the reduced luminance range of 200 . The column on the left represents a condition of minimum luminance equal to $2 \mathrm{~cd} / \mathrm{m}^{2}$, while the right column represent a system calibrated with a minimum luminance of $4 \mathrm{~cd} / \mathrm{m}^{2}$.

we observed for the monochrome AMLCD where the contrast typically increased at high luminance and decreased at low luminance for off-normal viewing directions.

\section{DISCUSSION}

Our results show that the most significant changes in luminance and contrast as a function of viewing angle for the monochrome AMLCD (increased luminance and reduced contrast) occur in the low luminance region. The data also demonstrate that for the color AMLCD, the deviation from the expected GDF occurs at both ends of the luminance scale. This suggests that for a particular LCD technology, an appropriate selection of the luminance range over which the device operates might result in improved angular response. We note that the choice of luminance range is also limited by the absolute value of the minimum luminance, which de- 


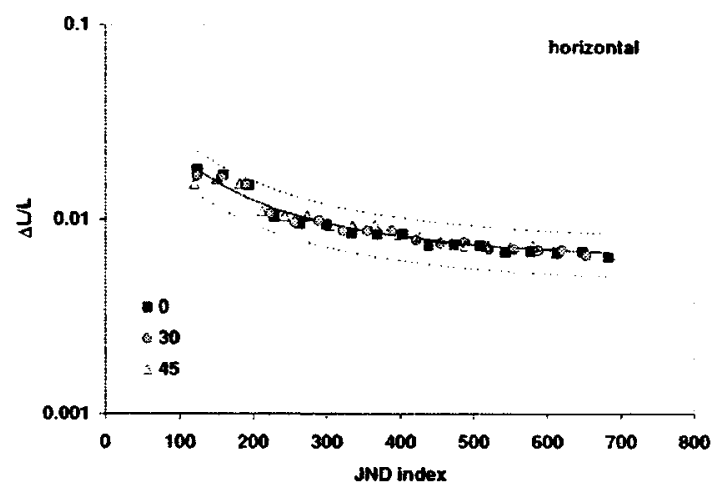

(a)

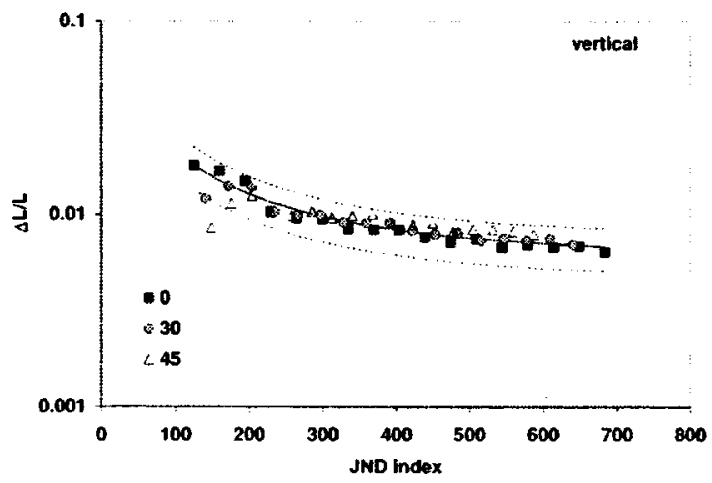

(c)

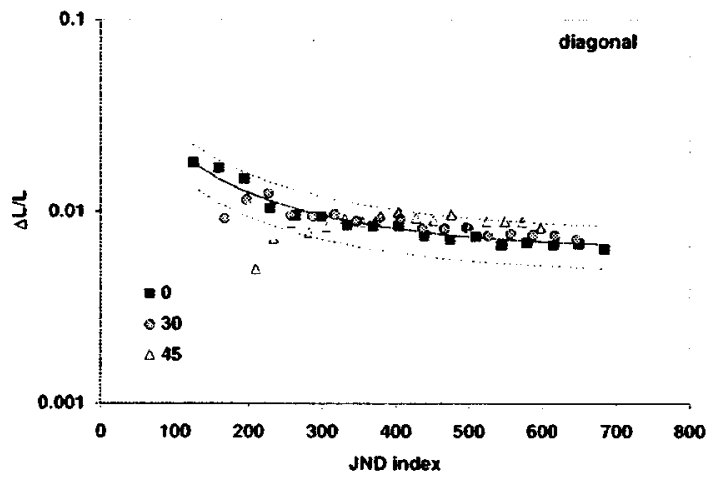

(e)

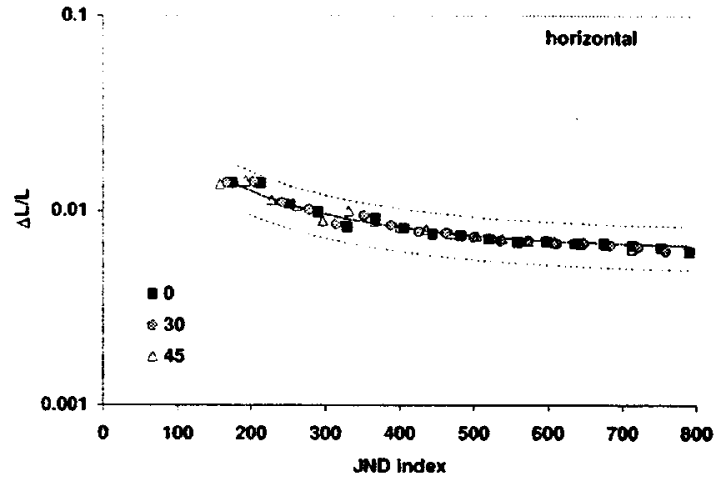

(b)



(d)

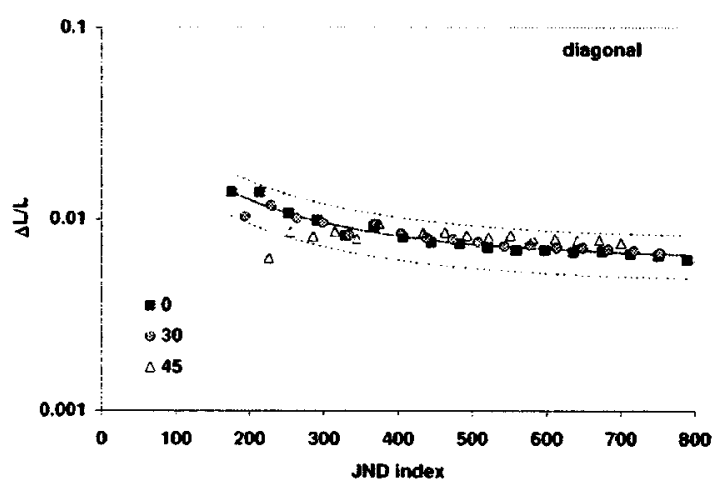

(f)

FIG. 9. The same data presented in Fig. 8, analyzed in terms of normalized contrast per JND. Again, the two columns correspond to the two luminance levels utilized for the reduced luminance range of 200 . The column on the left represents a condition of minimum luminance equal to $2 \mathrm{~cd} / \mathrm{m}^{2}$, while the right column represents a system calibrated with a minimum luminance of $4 \mathrm{~cd} / \mathrm{m}^{2}$. (see inserted text for details).

pends on the ambient illumination of the room where the displays will be used, and of the achievable maximum luminance for the specific monitor.

The results presented in Table I show clearly that the CRT luminance emission resembles the ideal Lambertian profile since the luminance reduction factor $L_{f}$ is between 0.88 and
0.91. Moreover, the results in Table I show that AMLCD emissions are far from Lambertian, with $L_{f}$ as large as 2.71 and as small as 0.60 for the angles and directions considered.

The calibration of a non-Lambertian display device depends on the acceptance angle of the luminance probe used to capture the luminance response across the grayscale. In 




(a)

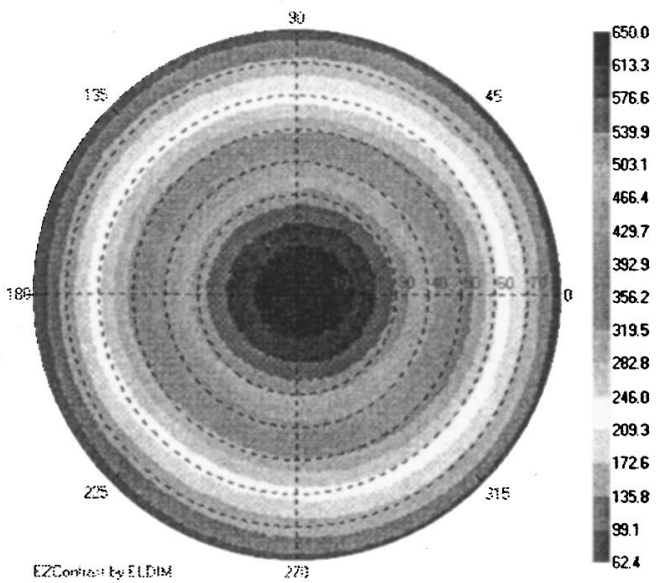

(b)

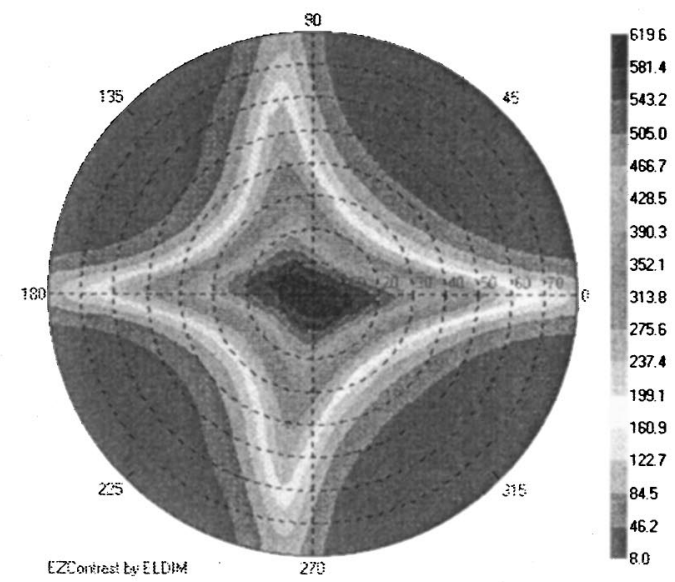

(c)

FIG. 10. Polar representation of the angular luminance results measured with the Fourier optics system for the medical AMLCD in the extended luminance range regime. Plots (a) and (b) represent the variation in luminance for the minimum and maximum luminance level, respectively. The ratio of plot (a) and (b) yields a contrast ratio metric, shown in (c).

this work, the grayscale calibration was done with the detector normally used for medical CRTs. The measurement of angular luminance was done with the collimated probe which has an acceptance angle of $1.5^{\circ}$, which might not correspond to the acceptance angle of the probe used for calibration. However, the dissimilar acceptance angles of the probes do not have any impact on the variation of luminance and contrast investigated in this study. Our goal is to measure the departure from the desired GDF achieved through a typical calibration procedure. The precision and accuracy of the display system to represent a given GDF for a given calibration technique is beyond the scope of this paper.

The two methods employed in this work to measure angular luminance curves are overall consistent in their results (see Fig. 11). The small discrepancies between the two methods are seen in all three angular directions represented in Fig. 11 in the low luminance region, suggesting that the bias in measured luminance is independent of the viewing angle. A possible explanation of why the two methods do not provide consistent low luminance measurements can be that luminance measurements of non-Lambertian surfaces are sensitive to the acceptance angle of the luminance meter device. The acceptance angles of the two methods used in this work are not equal. On one hand, the collimated probe has a welldefined acceptance angle of $1.5^{\circ}$. On the other hand, the acceptance angle of the Fourier optics method cannot be clearly defined since it is affected by the lens flare, ${ }^{21}$ and by optical scattering processes within the optical relay system. The acceptance angle for the Fourier system has not been measured during this work.

The clinical importance of our findings has not yet been reported and is not well understood. However, it is clear that the changes that occur at different viewing directions affect the visibility of lesions. Consider the case of a single user of 


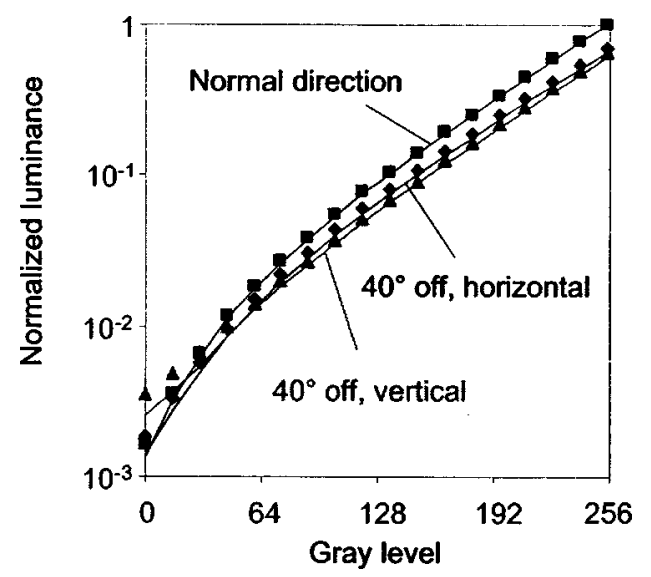

FIG. 11. Comparison of results from the two methods used to measure viewing angle in this paper. The data points correspond to measurements carried out with the collimated probe and rotating arm, while the continuous lines represent the data obtained with the Fourier optics system. For comparison, the results from the two methods are normalized to have the same luminance value for the maximum gray level in the normal direction.

the display device that will experience its effect when looking at different areas in the display screen, depending on the dimension of the screen surface (which can reach more than $30 \mathrm{~cm}$ in one of the sides). In this scenario, the more severe

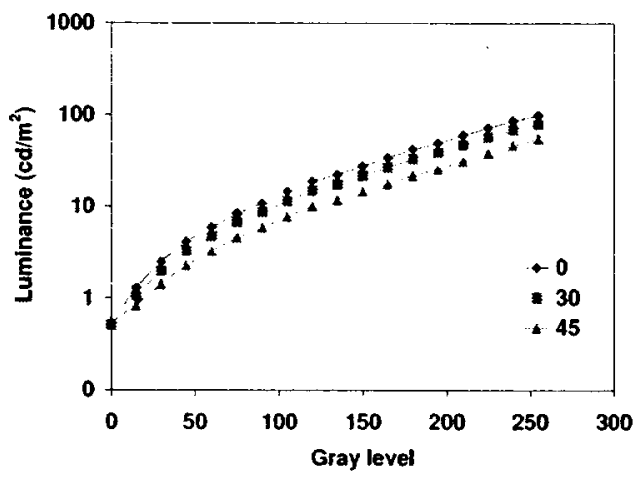

(a) changes in luminance and contrast associated with viewing angle are likely to happen at the corners of the screen. Alternatively, when two or more individuals are reading the same image displayed in the same screen, the departure from the on-axis calibration will be larger than in the previous case because larger angles are involved. This also applies to multi-monitor workstations that can have up to ten AMLCDs in a tiled arrangement.

Another aspect of these changes that adds complexity to the problem is that the changes affect the signal to be detected, as well as the background noise and anatomical structures present in the region of interest. For example, the reduction in contrast in low luminance regions described in this work for the medical AMLCD might result in a reduction in the contrast of a pulmonary nodule, but at the same time, the structural noise will be displayed with less contrast. Therefore, the net effect of this contrast reduction due to viewing angle is not obvious, and requires further investigation including psychophysics experiments with human and mathematical observers. ${ }^{22}$

\section{CONCLUSIONS}

We show that the emission from AMLCDs is far from Lambertian causing a reduction in image contrast at low lu-

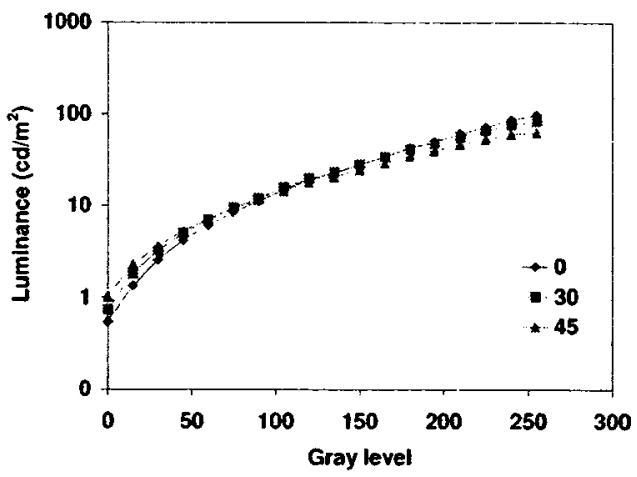

(b)

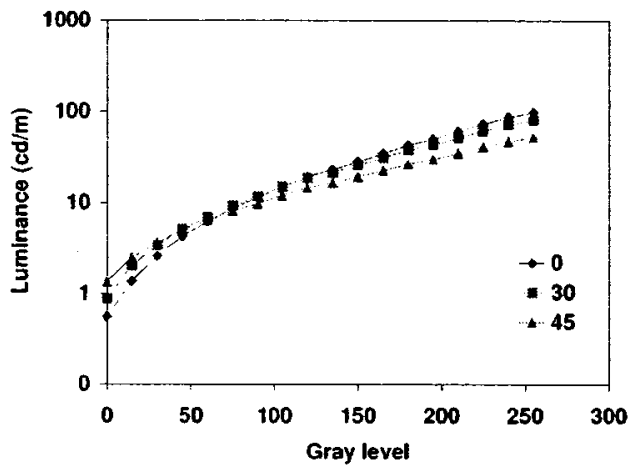

(c)

FIG. 12. Angular luminance profiles for the color AMLCD. The three plots show the luminance output as a function of gray level for selected off-normal angles in the horizontal (a), vertical (b), and diagonal (c) directions. 


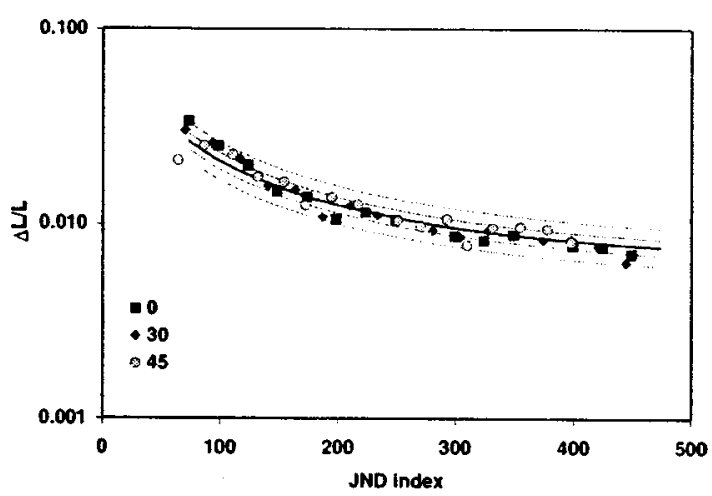

(a)

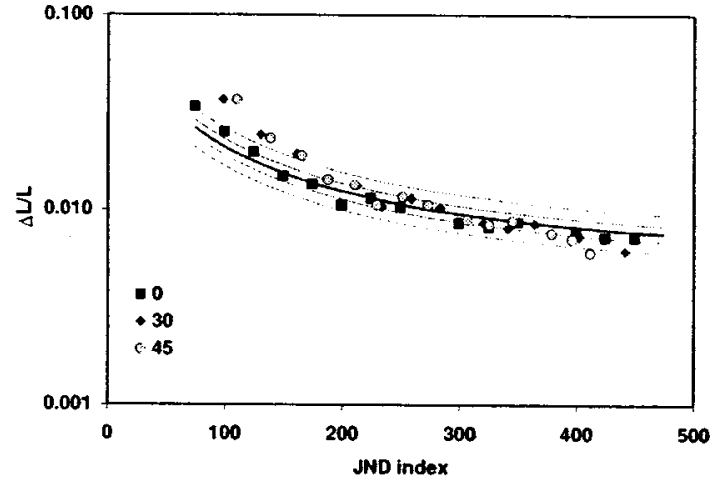

(b)

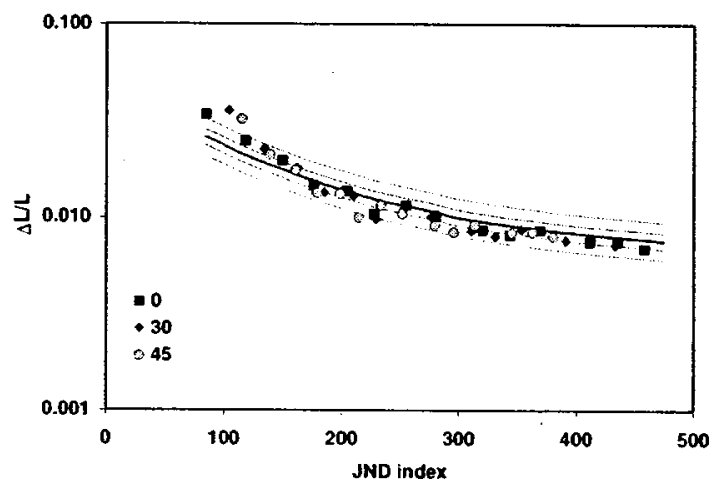

(c)

FIG. 13. The same data presented in Fig. 12, presented in terms of normalized contrast per JND, in the horizontal (a), vertical (b), and diagonal (c) directions.

minance levels, and a reduction of the available luminance range. We show also that this departure from the desired GDF is not observed in CRTs. For the monochrome AMLCD display, we find that the viewing angle characteristics in the horizontal direction are better than those along the vertical direction, and that the contrast response is worse along the diagonal directions. We conclude also from our analysis that the changes in luminance and therefore in contrast are most important in areas of low luminance. Particularly, we show that the minimum luminance of the monochrome AMLCD changes by a factor of 10 when viewing the display from the diagonal viewing directions. Finally, we show that color AMLCDs for desktop applications with a more modest luminance range can exhibit a relatively better angular response. Understanding of how display luminance and contrast are affected by the observer's viewing direction permits the investigation of the effect that viewing angle performance of display devices has on diagnostically relevant visual tasks.

\section{ACKNOWLEDGMENTS}

The authors thank Susan J. Hipper (Marquette University, Milwaukee, WI) for performing luminance measurements, and the Army Research Laboratory (Adelphi, MD) for pro- viding assistance with the ELDIM system. The authors acknowledge DOME Imaging Systems, Inc. and CEMAXICON for equipment loans.

${ }^{a)}$ Electronic mail: agb@cdrh.fda.gov

${ }^{1}$ M. J. Flynn, J. Kanicki, A. Badano, and W. R. Eyler, "High fidelity electronic display of digital radiographs," Radiographics 19, 1653-1669 (1999).

${ }^{2}$ A. Badano, "Modeling the bidirectional reflectance of emissive displays," Appl. Opt. 41, 3847 (2002).

${ }^{3}$ S.-J. Lee, A. Badano, and J. Kanicki, "Optical modeling of organic polymer light-emitting devices on flexible plastic substrates by a Monte Carlo method," in Proceedings Of the International Symposium on Optical Science and Technology SPIE's 47th Annual Meeting, SPIE, 2002.

${ }^{4}$ Y. Iimura and S. Kobayashi, "Electro-optical characteristics of amorphous and super-multidomain TN-LCDs prepared by a non-rubbing method," Proceedings of the Society for Information Display, 1994, pp. 915-918.

${ }^{5}$ N. Koma, Y. Baba, and K. Matsuoka, "No-rub multi-domain TFT-LCD using surrounding-electrode method," Proceedings of the Society for Information Display, 1995, pp. 869-872.

${ }^{6}$ J. Chen et al., "Four-domain TN-LCD fabricated by reverse rubbing or double evaporation," Proceedings of the Society for Information Display, 1995, pp. 865-868.

${ }^{7}$ M. Schadt, H. Sieberle, and A. Schuster, "Optical patterning of multidomain LCD with wide viewing angle,' Nature (London) 381, 212-215 (1996).

${ }^{8}$ M. S. Nam et al., "Wide-viewing-angle TFT-LCD with photo-aligned four-domain TN mode," Proceedings of the Society for Information Display, 1997, pp. 933-936. 
${ }^{9}$ J. Chen et al., "Optical simulation of electro-optical performance of low$\delta$ nd multi-domain TN displays," Proceedings of the Society for Information Display, 1997, pp. 937-940.

${ }^{10}$ Y. Masutani et al., "Novel TFT-array structure for LCD monitors with in-plane switching mode," Proceedings of the Society for Information Display, 1997, pp. 15-18.

${ }^{11} \mathrm{H}$. Wakemoto et al., "An advanced in-plane switching mode TFT-LCD," Proceedings of the Society for Information Display, 1997, pp. 929-932.

${ }^{12} \mathrm{~K}$. Ohmuro et al., "Development of super-high-image-quality verticalalignment-mode LCD," Proceedings of the Society for Information Display, 1997, pp. 845-848.

${ }^{13}$ C. D. Hoke, H. Mori, and P. J. Bos, "An ultra-wide-viewing angle STNLCD with a negative-birefringence compensation film," International Display Research Conference, 1997, pp. 21-24.

${ }^{14} \mathrm{H}$. Mori and P. J. Bos, "Application of a negative birefringence film to various LCD modes," International Display Research Conference, 1997, pp. M88-M97.

${ }^{15} \mathrm{~K}$. Schleupen et al., "High-information-content color 16.3 in.-desktopAMLCD with 15.7 million $a$-Si:H TFTs," International Display Research Conference, 1998, pp. 187-190.

${ }^{16}$ A. Badano and M. J. Flynn, "A method for measuring veiling glare in high performance display devices," Appl. Opt. 39, 2059-2066 (2000).
${ }^{17}$ National Electrical Manufacturers Assoc., "Digital Imaging and Communications in Medicine (DICOM), Part 3.14, Grayscale Standard Display Function," Technical report, ACR/NEMA, January 1998.

${ }^{18}$ E. Samei, A. Badano, D. Chakraborty, K. Compton, C. Cornelius, K. Corrigan, M. J. Flynn, B. Hemminger, N. Hangiandreou, J. Johnson, M. Moxley, W. Pavlicek, H. Roehrig, L. Rutz, J. Shepard, R. Uzenoff, J. Wang, and C. Willis, "Assessment of Display Performance for Medical Imaging Systems. Draft Report of the American Association of Physicists in Medicine Task Group 18, Version 9.1," American Association of Physicists in Medicine, 2003.

${ }^{19}$ B. E. A. Saleh and K. Lu, "The Fourier scope: An optical instrument for measuring LCD viewing-angle characteristics," J. SID 4, 33-40 (1996).

${ }^{20} \mathrm{M}$. Ohta, K. Kondo, and M. Oh-e, "Wide viewing-angle displays with in-plane switching mode of nematic LCs addressed by TFTs," Institute of Electronics, Information and Communication Engineers of Japan [Trans. Electron. E79-C, 1069-1075 (1996)].

${ }^{21} \mathrm{~S}$. Matsuda and T. Nitoh, "Flare as applied to photographic lenses," Appl. Opt. 11, 1850-1856 (1972).

${ }^{22}$ A. Badano, B. D. Gallas, K. J. Myers, and A. E. Burgess, "Effect of viewing angle on visual detection in liquid crystal displays," in Proceedings of Medical Imaging, Vol. 5029, SPIE, 2003. 